\title{
Continuous positive airway pressure does not reduce blood pressure in nonsleepy hypertensive OSA patients
}

\author{
G.V. Robinson, D.M. Smith, B.A. Langford, R.J.O. Davies and J.R. Stradling
}

ABSTRACT: Obstructive sleep apnoea (OSA) is associated with high cardiovascular morbidity and mortality. Several randomised controlled trials have shown that continuous positive airway pressure (CPAP) treatment of OSA reduces blood pressure (BP). This randomised, sham-placebo controlled crossover trial assesses whether CPAP produces a similar clinically significant fall in BP in hypertensive OSA patients, but without hypersomnolence.

Thirty-five, nonsleepy, hypertensive patients with OSA were treated with CPAP for 1 month, randomised first to either therapeutic or sham-placebo (subtherapeutic CPAP, about $1 \mathrm{cmH}_{2} \mathrm{O}$ pressure). The second months' alternative treatment followed a 2-week washout period. BP was measured over $24 \mathrm{~h}$, before and at the end of the two treatment periods: mean 24-h BP was the primary outcome variable.

There was no overall significant difference in mean 24-h BP: the change in mean 24-h BP on therapeutic CPAP was $\mathbf{- 2 . 1} \mathrm{mmHg}$ (SD 8.1), and $\mathbf{- 1 . 1} \mathrm{mmHg}$ (SD 8.1) on subtherapeutic CPAP, with a difference of $0.7 \mathrm{mmHg}(95 \%$ confidence interval $(\mathrm{Cl})+2.9-4.4)$. There was a small significant fall in Epworth Sleepiness Score, therapeutic (-1.4) versus sham (-0.3), and difference $-1.2(95 \% \mathrm{Cl}$ -2.0- -0.4), but no change in objective sleepiness.

In nonhypersomnolent hypertensive patients with obstructive sleep apnoea, there is no significant fall in mean 24-h blood pressure with continuous positive airway pressure, in contrast to the fall seen in hypersomnolent patients with obstructive sleep apnoea.

KEYWORDS: Cardiovascular risk, continuous positive airway pressure, hypertension, obstructive sleep apnoea

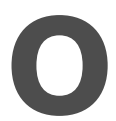
bstructive sleep apnoea (OSA) is common, with $2-4 \%$ of adult males, and about $1 \%$ of adult females having detectable sleep apnoea on overnight sleep study [1]. Of all UK males, $\sim 1 \%$ have moderate or severe disease, appropriate for treatment with nasal continuous positive airway pressure treatment (nCPAP) [2]. In OSA, recurrent upper airway closure causing transient asphyxia leads to sleep fragmentation with subsequent daytime hypersomnolence. Daytime hypersomnolence is associated with an increase in driving accidents [3] and poor quality of life [4]. Both day and nightime blood pressure (BP) are raised in OSA [5]. The raised BP is seen in community epidemiological [6] and hospital-based studies [7], and is independent of obesity (the commonest cause of OSA), and the other common risk factors for hypertension, which are also frequently present in this patient population.

nCPAP treatment is the most effective treatment for severe OSA [8]. Randomised controlled trials have shown improvement of daytime sleepiness with nCPAP [9-11], which is usually improved after the first night of treatment, and certainly after 2 weeks [12]. Recent randomised controlled trials have shown that CPAP treatment of severe OSA reduces 24-h BP [13-18]. In the Oxford randomised parallel controlled trial [13], there was a mean ambulatory BP fall of $3.3 \mathrm{mmHg}$ (95\% confidence interval (CI) -5.3- -1.3) with CPAP treatment relative to controls, with an even larger $\mathrm{BP}$ fall of $6.6 \mathrm{mmHg}$ in those on antihypertensive drugs. Patients with more severe disease ( $>30$ dips per hour in arterial oxygen saturation $\left(\mathrm{Sa}_{2} \mathrm{O}_{2}\right)$ of $\left.>4 \%\right)$ had greater falls in $\mathrm{BP}$, and the $\mathrm{BP}$ reduction appeared to be independent of baseline BP. The mechanism for the hypertension of OSA is uncertain, but is likely to be related to increased sympathetic tone, and catecholamine excretion [19]. Increased sympathetic tone may result from recurrent nocturnal hypoxia (or hypercapnia), sleep fragmentation, or greater pleural pressure falls due to the increased

\section{AFFILIATIONS}

Oxford Centre for Respiratory Medicine, Oxford Radcliffe Hospitals NHS Trust, Churchill Hospital Site, Oxford, UK.

\section{CORRESPONDENCE}

G.V. Robinson

Oxford Centre for Respiratory Medicine

Oxford Radcliffe Hospitals NHS Trust Churchill Hospital Site

Oxford

0X3 7LJ

UK

Fax: 441865225221

E-mail: gracevrobinson@

yahoo.co.uk

Received

May 302005

Accepted after revision:

January 202006

SUPPORT STATEMENT

The British Heart Foundation (study number PG/01/171/13395) provided funding 
inspiratory effort which develops during hypopnoeas and apnoeas [20].

Nasal CPAP is currently used to improve the daytime hypersomnolence of OSA [21]. Depending on the definition used, $1-5 \%$ of UK adult males have detectable sleep apnoea on sleep study [1], with $\sim 1 \%$ having sufficient daytime symptoms to warrant treatment with CPAP [2]. Therefore, perhaps up to $4 \%$ of UK adults have OSA that is detectable on sleep study, but without sufficient excess hypersomnolence to warrant CPAP therapy.

Due to the high incidence of cardiovascular complications in patients with OSA, some now advocate CPAP treatment for all these patients [22], regardless of daytime symptoms. The average patient with OSA has a 10-yr cardiovascular risk of $\sim 30 \%$ (myocardial infarction and stroke risk combined), predicted from conventional risk factors [23]. From large prospective studies, a BP fall of $3.3 \mathrm{mmHg}$ would be expected to be associated with a $20 \%$ stroke and $15 \%$ coronary heart disease event rate risk reduction [24]; thus the BP falls seen with CPAP treatment have significant potential reductions in vascular risk. At present there is no data to support the extension of treatment to all-comers with only sleep-study proven OSA in the absence of daytime sleepiness, and, as a result, there is the potential to waste considerable resources.

This trial aimed to assess whether CPAP treatment produces a clinically significant fall in 24-h BP in hypertensive patients with OSA, of similar magnitude to that seen in symptomatic patients, but without sufficient daytime symptoms to warrant CPAP therapy. This would establish if there is likely to be a cardiovascular benefit to treating hypertensive patients with OSA, even when they do not have significant daytime hypersomnolence. The study was not designed to determine the aetiology of any such BP fall. The aim was to answer the simple clinical question as to whether there is a BP advantage to treating nonhypersomnolent OSA patients.

\section{METHODS}

\section{Design and setting}

A randomised sham-placebo controlled, cross-over study of patients attending the Sleep and Respiratory Trials Unit, Oxford Centre for Respiratory Medicine, Oxford, UK, was performed. The unit is a regional referral centre and the majority of referrals are for possible OSA. About one-third of patients are from the immediate Oxford area. Referrals are made from general practitioners $(36 \%)$, ear, nose and throat surgeons $(41 \%)$, and other hospital consultants $(23 \%)$.

\section{Patients}

Patients were eligible for the trial if they were aged $>18 \mathrm{yrs}$, had proven obstructive sleep apnoea with $>10$ dips in $\mathrm{Sa}_{2} \mathrm{O}_{2}$ of $>4 \%$ per hour on overnight sleep study, and had no daytime hypersomnolence, with an Epworth Sleepiness Score (ESS) $<10$ [25]. Subjects fulfilling these two criteria were then assessed for hypertension. This was defined as either taking antihypertensive drugs, or a BP $>140 / 90 \mathrm{mmHg}$ on 24 -h ambulatory BP monitoring. The 24-h BP recording was only performed on potential subjects if a casual clinic BP was $>140 / 90 \mathrm{mmHg}$, and if they were not already on antihypertensive drugs. Thus, hypertensive patients were recruited who had significant sleep study OSA, but were without sufficient daytime hypersomnolence to warrant CPAP treatment.

Patients were excluded if they had respiratory failure, declined to participate, or were unable to give informed consent. A previous cardiovascular event, or the presence of any additional cardiovascular risk factors, was not a determinant in offering or declining a patient entry to the trial. The study was approved by the Central Oxford Research Ethics Committee (C01.218), and all participants gave written informed consent.

\section{Procedures}

Obstructive sleep apnoea was diagnosed from a one-night respiratory polysomnographic study, and has been described before [13]. In brief, patients' body movements, heart rate rises and transient pulse transit time (PTT) falls (BP rises) were recorded as measures of "autonomic arousal" from sleep. The PTT signal and body movements recorded on video are robust markers of arousal and, along with $\mathrm{Sa}_{2} \mathrm{O}_{2}$, snoring and increases in the respiratory swing in PTT, are accurate in diagnosing and quantifying OSA severity [26] (Win-Visi monitoring system, Stowood Scientific Instruments, Oxford, UK). The results of the sleep study are scored automatically, with manual review to ensure data accuracy. OSA was diagnosed from a review of all data, including the video recording.

The severity of sleep apnoea was then quantified numerically as the number of dips in $\mathrm{Sa}_{1} \mathrm{O}_{2}$ of $>4 \%$ for every hour of the study. This index is one of the best predictors of response to nCPAP [27], correlates well with conventional apnoea/ hypopnoea index (AHI) measurements, and is the most consistent index between repeat studies of patients with OSA [28].

The 24-h BP was measured with validated ambulatory recorders (TM 2420 or TM 2421 (Takeda A\&D, Tokyo, Japan)) [29]. A trained nurse fitted an appropriately sized cuff on the patients' nondominant arm, which was worn for the subsequent $24 \mathrm{~h}$ during normal daily activities. Monitors were programmed to record BP every $30 \mathrm{~min}$, and subjects were instructed to switch the machine off whilst driving. Patients completed a diary card, and pressed the event marker to identify sleeping and wakeful periods. When two or more readings occurred within the same half hour (due to pressing the event marker), these were averaged to give one recording for that time period.

Patients assessed their subjective daytime sleepiness using the ESS, a self-completed questionnaire quantifying the tendency to fall asleep in various daytime situations [25]. Objective sleepiness was measured with the Osler test (Stowood Scientific Instruments, Oxford, UK), a behavioural maintenance of wakefulness test (MWT), which assesses the subjects' ability to remain awake in a dark and sound-isolated room for up to $40 \mathrm{~min}$ [30]. Even though patients were selected for the absence of subjective daytime symptoms, it was expected that a number of subjects might be sleepy on objective measurements.

Therapeutic nCPAP was generated using an automatic CPAP machine (Autoset Spirit, Resmed, Abingdon, UK) [31]; the control arm used a fixed, subtherapeutic pressure from the same machine, as previously described [13]. Briefly, the shamplacebo (subtherapeutic) CPAP machine delivers $<1 \mathrm{cmH}_{2} \mathrm{O}$ 
pressure, which is insufficient to hold open the pharynx. In all other respects, sham-placebo CPAP is identical to therapeutic CPAP. Patients were not aware which CPAP pressure they had received, and the nurse who assigned the patients to each treatment arm did not take part in outcome assessments. The investigators who assessed the study outcomes were not involved in randomisation or patient CPAP set-up. A crossover design was chosen, as a therapeutic effect on symptoms that might un-blind the patients was not expected.

Patients received the standard CPAP induction programme. Following baseline assessments, patients were randomised to the first treatment arm (either therapeutic or subtherapeutic CPAP) for 1 month. Randomisation was by a series of presealed and numbered opaque envelopes. The second randomised treatment period followed a 2-week washout period. The 24-h BP, and subjective and objective sleepiness were assessed before and after each treatment period. CPAP compliance during both treatment periods and residual AHI following therapeutic CPAP were measured from the machines' internal microprocessor. It was not possible to measure AHI after subtherapeutic CPAP for technical reasons. Patients were instructed not to change their antihypertensive medication during the study period.

A specialist nurse team assisted patients with telephone advice for any CPAP difficulties during each treatment period, and masks were adjusted as necessary. At the end of the study, subjects were given the option to continue with CPAP long term. If they wished to continue, they were discharged with a fixed pressure machine, the pressure being derived from the study period on the therapeutic autoadjusting CPAP machine.

\section{Sample size}

The study size was calculated from the results of the present authors' previous randomised parallel trial assessing the effect of therapeutic and subtherapeutic CPAP on 24-h ambulatory BP [13]. Results of this study suggested that a difference in mean 24-h BP of $>5 \mathrm{mmHg}$ could be excluded with 26 patients crossing over (alpha $=0.05$, power $=90 \%$ ). Thus, allowing for a $15 \%$ dropout rate, a minimum of 30 patients had to be recruited. The data are presented according to current recommendations (CONSORT) [32].

\section{Data analysis}

The data are presented as mean $\pm \mathrm{SD}$, unless non-normally distributed, when they are presented as median (interquartile range (IQR)).

The primary end-point was change in mean BP ( $1 / 3$ systolic plus $2 / 3$ diastolic), averaged over $24 \mathrm{~h}$, after therapeutic or subtherapeutic CPAP. Secondary analyses included change in systolic and diastolic BP, and change in mean BP during awake and sleeping periods. Changes in BP were assessed with paired t-tests. The correlation between the change in BP and the two potential predictors, baseline BP and OSA severity, were examined, as was any correlation with therapeutic CPAP compliance.

\section{RESULTS}

Figure 1 shows the trial profile. Of the 40 patients eligible for the trial (with $>10$ dips in $\mathrm{Sa}_{1} \mathrm{O}_{2}$ of $>4 \%$ per hour on sleep study) with an ESS $<10$, five did not have hypertension on 24-h BP monitoring, leaving 35 eligible for the study. Twentyseven of these subjects had drug-treated hypertension; the other eight had hypertension confirmed on 24-h BP monitoring. Seven subjects were taking $\geqslant 2$ classes of antihypertensive drug; the other 20 were on a single drug. Similar numbers of patients were taking a $\beta$-blocker $(n=7)$, diuretic $(n=6)$, angiotensin-converting enzyme inhibitor or angiotensin II blocker $(n=8)$, or a calcium channel blocker $(n=5)$. One patient was taking an $\alpha$-blocker.

Eighteen patients received therapeutic CPAP, and 17 received subtherapeutic CPAP for the first month. Two of the patients treated in the therapeutic CPAP arm first were withdrawn before completing the first months' treatment period, one because of intolerance of the BP cuff, and one because the BP data collected were inadequate. Following the 2-week washout period, subjects were given the alternative treatment. Thus, in the second limb, 17 patients were treated with therapeutic $\mathrm{CPAP}$, and 16 were treated with subtherapeutic CPAP. One subject was withdrawn from the therapeutic CPAP arm during the second month because of intolerance of the BP cuff, leaving 32 patients completing both treatment arms of the study.

Table 1 shows the patients' characteristics at baseline. The subjects had significant sleep apnoea, with a median (IQR) of 28.1 (18-38) dips in $\mathrm{Sa}_{\mathrm{a}} \mathrm{O}_{2}$ of $>4 \%$ per hour, but no significant

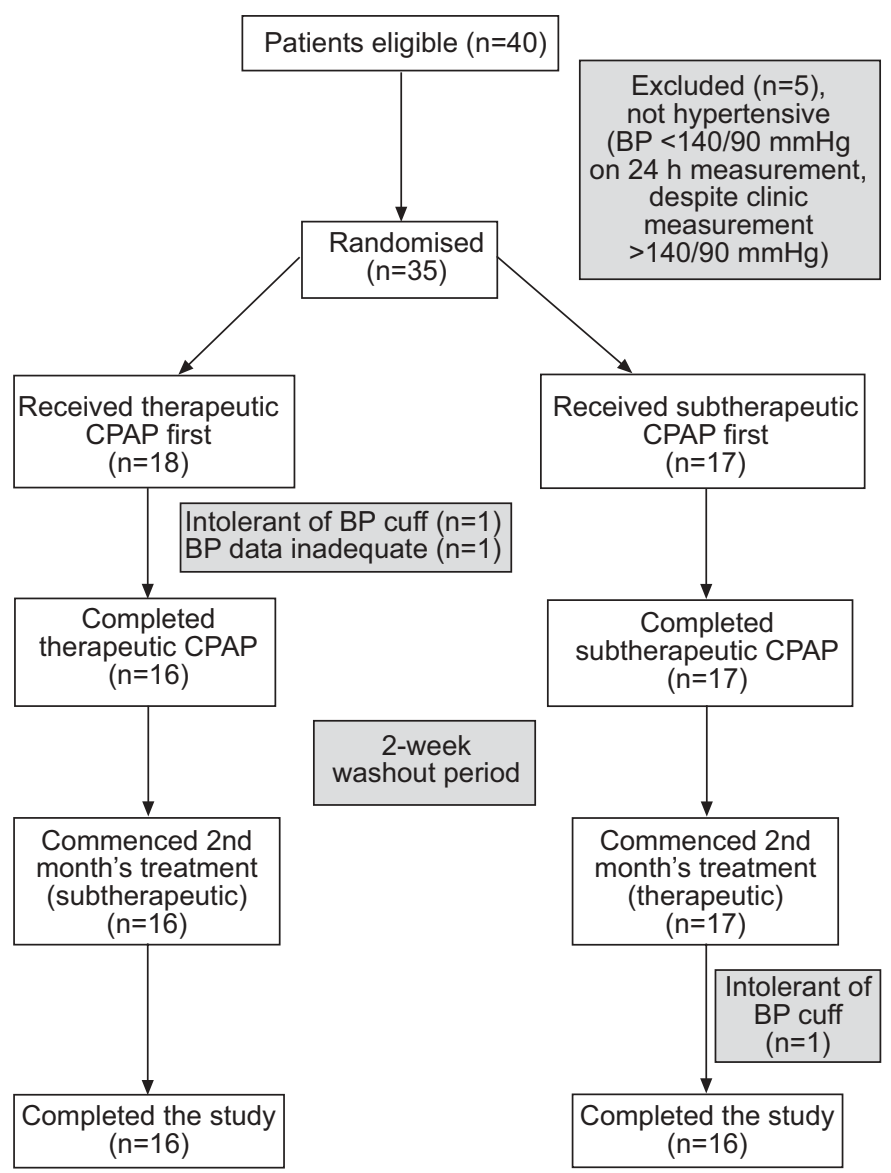

FIGURE 1. Trial profile. BP: blood pressure; CPAP: continuous positive airway pressure. 


\begin{tabular}{|c|c|}
\hline Age yrs & $54 \pm 8$ \\
\hline Male/female $\mathrm{n}$ & $31 / 4$ \\
\hline $\mathrm{BMI} \mathbf{k g} \cdot \mathrm{m}^{-2}$ & $33.2 \pm 5.3$ \\
\hline Neck size cm & $43.9 \pm 4.0$ \\
\hline ESS & $5.3(3.0-7.0)$ \\
\hline Osler test min & $40(40-40)$ \\
\hline Dips in $\mathrm{Sa}, \mathrm{O}_{2}$ of $>4 \%$ per hour of sleep $n$ & $28.1(18.0-38.0)$ \\
\hline Drugs/no drugs for hypertension $\mathrm{n}$ & $27 / 8$ \\
\hline \multicolumn{2}{|c|}{ 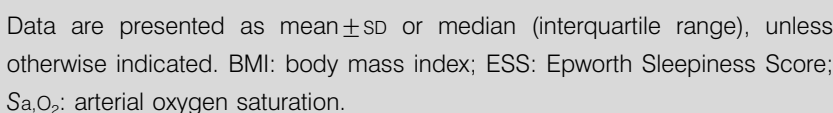 } \\
\hline
\end{tabular}

hypersomnolence, with a median baseline ESS of 5.3 (3.0-7.0), and a median baseline MWT of $40 \mathrm{~min}$ (40-40 $\mathrm{min}$ ).

Table 2 shows the BP data for the present study. There was no overall significant difference in mean 24-h BP. The change in mean $24-\mathrm{h}$ BP on therapeutic CPAP was $-2.1 \mathrm{mmHg}(-0.5$ during waking hours, -2.9 during sleeping hours), and $-1.1 \mathrm{mmHg}$ on subtherapeutic CPAP (-1.2 awake, +0.1 asleep), a difference of $0.7 \mathrm{mmHg}(95 \% \mathrm{CI}+2.9-4.4)$. There was no significant BP fall on therapeutic or subtherapeutic CPAP when the BP was divided into 24-h systolic and diastolic BP, or into sleeping and waking BP. Review of the Autoset CPAP microprocessor data showed resolution of oxygen desaturations with therapeutic CPAP (residual AHI 5.5 \pm 3.7 ).

There was a small, significant difference in mean CPAP compliance between the two treatment arms. Mean CPAP use on therapeutic CPAP was $5.2 \pm 2.1 \mathrm{~h}$ per night, and $4.3 \pm$ $2.4 \mathrm{~h}$ per night on subtherapeutic CPAP $(\mathrm{p}<0.001)$. This was presumed to be because subjects felt symptomatically better on therapeutic CPAP, leading to increased overnight use. This fits with the small, but significant, fall in ESS (subjective sleepiness) seen on therapeutic CPAP of -1.4 versus -0.3 on subtherapeutic CPAP $(\mathrm{p}<0.02)$. However there was no change in MWT $(\mathrm{p}>0.8)$, which was as expected this was essentially normal at baseline. Only four of the 32 subjects failed to reach the full $40 \mathrm{~min}$ on MWT prior to therapeutic CPAP, and five failed after treatment. Seven subjects failed to reach the full $40 \mathrm{~min}$ prior to subtherapeutic CPAP, and two failed after subtherapeutic CPAP treatment (all nonsignificant).
Excluding those subjects with a poor CPAP compliance $(<2 \mathrm{~h}$ per night), did not alter the BP data significantly. During the subtherapeutic CPAP treatment arm, six subjects had a compliance $<2 \mathrm{~h}$ per night, and during the therapeutic CPAP arm, three subjects had a compliance of $<2 \mathrm{~h}$ per night. These three subjects were also poor compliers on subtherapeutic CPAP. With the poor CPAP compliers excluded from the data, the change in mean $24-\mathrm{h} \mathrm{BP}$ was -2.0 and $-1.4 \mathrm{mmHg}$ on therapeutic and subtherapeutic CPAP, respectively (difference $-1.0,95 \% \mathrm{CI}+3.4--5.4$ ).

The median baseline dip rate in $\mathrm{Sa}_{1} \mathrm{O}_{2}$ of $>4 \%$ was 28.1 . There was no significant overall mean BP change on therapeutic or subtherapeutic CPAP depending on baseline OSA severity. For those in the bottom half of the group (defined as having a $>4 \%$ $\mathrm{Sa}_{\mathrm{a}} \mathrm{O}_{2}$ dip rate of $\leqslant 28.1$ ), the mean 24 -h BP change was -3.3 and $+0.5 \mathrm{mmHg}$ on therapeutic and subtherapeutic CPAP, respectively; a difference of $-3.8 \mathrm{mmHg}(95 \% \mathrm{CI}-9.6-+2.0)$. For those in the top half of the group (defined as having a $>4 \% \mathrm{Sa}_{1} \mathrm{O}_{2}$ dip rate of $\geqslant 28.1$ ), the change in mean $24-\mathrm{h}$ BP was -0.8 and $+2.9 \mathrm{mmHg}$ on therapeutic and subtherapeutic CPAP, respectively; a difference of $+1.5 \mathrm{mmHg}(95 \% \mathrm{CI}+8.4--5.4)$. There was no correlation between baseline OSA severity and BP fall, showing that even at the most severe end of the present study's patient population, there was no significant BP fall, as seen in previous studies with symptomatic patients [13-18].

At the end of the study period, all of the 32 subjects elected to continue with CPAP, but by 12 months this had dropped to 28 of $32(87.5 \%)$. The mean 95th centile titration pressure was $10.4 \pm 1.5 \mathrm{cmH}_{2} \mathrm{O}$. The main reason given by patients for continuing CPAP was the improvement in snoring on treatment.

\section{DISCUSSION}

This study has shown no overall difference in the primary endpoint of mean 24-h ambulatory BP in patients with moderateto-severe OSA, but without daytime hypersomnolence when treated with therapeutic CPAP. Further analysis has shown no change in systolic and diastolic $\mathrm{BP}$, and no change during sleep and wake periods. This is in contrast to the BP fall seen in hypersomnolent patients with OSA treated with CPAP [13-15, 17]. It also suggests that hypersomnolence might in some way be important for the pathogenesis of the hypertension of sleep apnoea. These data further suggest that there is unlikely to be a cardiovascular benefit to treating nonhypersomnolent

TABLE 2 Blood pressure (BP) results

\begin{tabular}{|c|c|c|c|c|c|c|}
\hline & \multicolumn{2}{|c|}{ Subtherapeutic CPAP } & \multicolumn{2}{|c|}{ Therapeutic CPAP } & \multirow[t]{2}{*}{ Difference in BP change ${ }^{\#}$} & \multirow[t]{2}{*}{ p-value } \\
\hline & Before & After & Before & After & & \\
\hline 24-h BP mmHg & $105.1 \pm 12.1$ & $103.9 \pm 12.7$ & $103.4 \pm 11.6$ & $101.4 \pm 12.5$ & $-0.74(-4.4-+2.9)$ & 0.71 \\
\hline Wake period BP $\mathrm{mmHg}$ & $108.8 \pm 13.0$ & $107.6 \pm 13.7$ & $106.1 \pm 13.6$ & $105.6 \pm 13.2$ & $+1.10(-2.9-+5.1)$ & 0.61 \\
\hline Sleep period BP $\mathrm{mmHg}$ & $98.0 \pm 14.8$ & $97.8 \pm 13.6$ & $96.0 \pm 11.5$ & $93.3 \pm 12.3$ & $-2.81(-7.8-+2.2)$ & 0.31 \\
\hline 24-h systolic BP mmHg & $143.0 \pm 17.3$ & $139.3 \pm 17.6$ & $140.3 \pm 16.1$ & $137.0 \pm 16.3$ & $-0.10(-5.3-+5.1)$ & 0.87 \\
\hline 24-h diastolic BP $\mathrm{mmHg}$ & $86.7 \pm 11.1$ & $86.8 \pm 11.6$ & $85.3 \pm 11.2$ & $84.2 \pm 11.7$ & $-1.47(-6.7-+3.8)$ & 0.59 \\
\hline
\end{tabular}

Data are presented as mean \pm SD, unless otherwise indicated. ${ }^{\#}$ : median (95\% confidence interval), negative figures=therapeutic CPAP lower; ${ }^{\bullet}$ : p-value of the difference of the differences. CPAP: continuous positive airway pressure. 
hypertensive patients with OSA, through reducing 24-h BP (despite their high baseline cardiovascular risk), as has been suggested by some [22].

The potential limitations of this study include the likely heterogeneous population of subjects studied, in terms of the cause of their hypertension. There might be a subgroup of patients on particular antihypertensive drugs that behave differently. To answer this question will need a much larger study. The treatment period was short, but comparable to the treatment period in the study by PEPPERELL et al. [13] (to aid data comparison), where treatment with subtherapeutic CPAP in hypersomnolent patients for $>1$ month would have been unethical. It is possible that a longer period of treatment might have led to an eventual BP fall.

The mechanism for the sustained rise in BP with OSA is uncertain, but is likely to be secondary to increased sympathetic tone and catecholamine excretion [20]. The cause of the increased sympathetic tone is not established, but this data could suggest that it may be indirectly related to the hypersomnolence, with both perhaps due to sleep fragmentation. Hypoxia, hypercapnia and pleural pressure fluctuation are also possible alternative causes $[33,34]$, but the absence of a $\mathrm{BP}$ fall in this patient group (despite resolution of these consequences), indirectly suggests that sleep fragmentation may be the more important mechanism.

Hypersomnolence is a marker of sleep fragmentation, so it follows that this patient population may have had less severe sleep fragmentation than in the previous studies on hypersomnolent patients [13-15, 17]. REES et al. [35] have shown considerable interindividual variation in the degree to which patients with OSA experience cortical electroencephalographic (EEG) arousal with each apnoea. It is possible that the previously studied patients included a population more susceptible to the cortical effects of apnoeas, with a higher proportion of apnoeas leading to EEG arousal, and hence causing sleep fragmentation and daytime hypersomnolence.

This current group of subjects had a median $>4 \% \mathrm{Sa}_{2} \mathrm{O}_{2}$ dip rate of 28.1. The present authors' previously studied OSA population had a baseline median $>4 \% \mathrm{Sa}_{1} \mathrm{O}_{2}$ dip rate of 33 [13]. In these earlier subjects, the majority of the BP falls with therapeutic CPAP was in those subjects with $>33$ dips in $\mathrm{Sa}_{\mathrm{a}} \mathrm{O}_{2}$ of $>4 \%$ per hour. In the currently studied population, there was no relationship between baseline OSA severity and BP fall. Subjects with the most severe OSA (the top 50\% of the group, i.e. those with $>28.1>4 \% \mathrm{Sa}_{1} \mathrm{O}_{2}$ dips) also showed no fall in $\mathrm{BP}$, indicating no dependence on the baseline dip rate in $\mathrm{Sa}_{1} \mathrm{O}_{2}$ of $>4 \%$ per hour. This confirms that it is unlikely that an effect seen only at the severe end of the spectrum has been missed.

The studies by PHILIPSON and co-workers $[36,37]$ using a dog model of OSA suggest that recurrent intermittent hypoxia is the more likely mechanism for the sustained hypertension of OSA, rather than sleep fragmentation [38], which is the more likely explanation for the transient BP rises seen following each apnoea. Studies in rats [39] also suggest that hypoxia may be the causal link, although BP elevation is seen in only some rat strains after exposure to chronic episodic hypoxia for 35 days. In these studies, the BP remained abnormally elevated for some time, in the absence of ongoing hypoxia; with evidence of adrenergic and renin-angiotensin system over-activity as mechanisms for the BP rise. No human studies have thus far addressed this issue. The current data suggests that hypoxia may not be the linking mechanism, but that an alternative hypothesis, recurrent arousals sufficient to produce daytime sleepiness, may be possible.

This current data is in accordance with that of BARBE et al. [16], who did not show a BP fall (24-h ambulatory measurements) with therapeutic CPAP after 6 weeks' treatment compared with sham (subtherapeutic) CPAP. This multicentre Spanish trial studied a more severe group of patients (mean AHI 55), with no hypertension or daytime hypersomnolence (mean ESS 7.0). No change in subjective or objective sleepiness measures were seen in this study.

BECKER et al. [14] and FACCENDA et al. [15] showed significant BP falls with CPAP compared with placebo (either sham CPAP or tablet, respectively), but the subjects studied were hypersomnolent (median ESS $\sim 14.2$ and $\sim 15$, respectively). BECKER et al. [14] studied more severe patients, with a mean AHI of $\sim 63$; the subjects of the study by FACCENDA et al. [15] had a mean AHI of 35 , similar to the patients studied in the present study.

The literature suggests that the greatest BP falls with CPAP are seen in those patients with hypersomnolence who are taking antihypertensive drugs [13-15]. Despite $77 \%$ of the present subjects being on antihypertensive drugs, there was no fall in $\mathrm{BP}$, further suggesting that a fall in BP should have been seen if nonhypersomnolent patients with OSA behave similarly to hypersomnolent patients.

Thus, although the power of this study in nonsleepy patients with OSA was limited, the present authors believe that the previous literature would have predicted a much larger BP fall with CPAP in these relatively severe, largely compliant, hypertensive patients mostly on antihypertensive medications; this was not seen in this study.

\section{Conclusion}

This study in hypertensive patients with obstructive sleep apnoea, but without daytime hypersomnolence, has not demonstrated a significant blood pressure fall with continuous positive airway pressure therapy, as would have been predicted from the available literature on patients with hypersomnolence. This suggests that sleep fragmentation may be important in the pathogenesis of hypertension in human sleep apnoea as well as hypersomnolence. If this is the case, then this potential link needs further exploration using more sophisticated measures of arousal and sleep fragmentation, in a range of both hypersomnolent and nonhypersomnolent patients. At present, based on the present study and that of BARBE et al. [16], treating nonhypersomnolent patients with OSA for potential effects on blood pressure, and therefore possibly cardiovascular risk, cannot be supported.

\section{REFERENCES}

1 Young T, Palta M, Dempsey J, et al. The occurrence of sleep-disordered breathing among middle-aged adults. $N$ Engl J Med 1993; 328: 1230-1235. 
2 Stradling JR, Barbour C, Glennon J, et al. Prevalence of sleepiness and its relation to autonomic evidence of arousals and increased inspiratory effort in a community based population of men and women. J Sleep Res 2000; 9: 381-388.

3 George CF, Nickerson PW, Hanly PJ, et al. Sleep apnoea patients have more automobile accidents. Lancet 1987; 2: 447.

4 Engleman HM, Martin SE, Deary IJ, et al. Effect of CPAP therapy on daytime function in patients with mild sleep apnoea/hypopnoea syndrome. Thorax 1997; 52: 114-119.

5 Peppard PE, Young T, Palta M, et al. Prospective study of the association between sleep-disordered breathing and hypertension. N Engl J Med 2000; 342: 1378-1384.

6 Nieto FJ, Young TB, Lind BK, et al. Association of sleepdisordered breathing, sleep apnea, and hypertension in a large community-based study. Sleep Heart Health Study. JAMA 2000; 283: 1829-1836.

7 Davies CWH, Crosby JH, Mullins RL, et al. Case-control study of 24 hour ambulatory blood pressure in patients with obstructive sleep apnoea and normal matched control subjects. Thorax 2000; 55: 736-740.

8 Sullivan CE, Issa FG, Berthon-Jones M, et al. Reversal of obstructive sleep apnoea by continuous positive airway pressure applied through the nares. Lancet 1981; 1: 862-865.

9 Engleman HM, Martin SE, Kingshott RN, et al. Randomised controlled trial of daytime function after continuous positive airway pressure (CPAP) therapy for the sleep apnoea/hypopnoea syndrome. Thorax 1998; 53: 341-345.

10 Engleman HM, Kingshott RN, Wraith PK, et al. Randomised placebo-controlled crossover trial of continuous positive airway pressure for mild sleep apnea/ hypopnea syndrome. Am J Respir Crit Care Med 1999; 159: 461-467.

11 Jenkinson C, Davies RJ, Mullins R, et al. Comparison of therapeutic and sub-therapeutic nasal continuous positive airway pressure for obstructive sleep apnoea: a randomised prospective parallel trial. Lancet 1999; 353: 2100-2105.

12 Lamphere J, Roehrs T, Wittig R, et al. Recovery of alertness after CPAP in apnea. Chest 1989; 96: 1364-1367.

13 Pepperell JCT, Ramdassingh-Dow S, Crosthwaite N, et al. Ambulatory blood pressure after therapeutic and subtherapeutic continuous positive airway pressure for obstructive sleep apnoea: a randomised controlled trial. Lancet 2002; 359: 204-209.

14 Becker HF, Jerrentrup A, Ploch $\mathrm{T}$, et al. Effect of nasal continuous positive airway pressure treatment on blood pressure in patients with obstructive sleep apnea. Circulation 2003; 107: 68-73.

15 Faccenda JF, Mackay TW, Boon NA, et al. Randomised placebo-controlled trial of continuous positive airway pressure on blood pressure in the sleep apnea-hypopnea syndrome. Am J Respir Crit Care Med 2001; 163: 344-348.

16 Barbe F, Mayoralas LR, Duran J, et al. Treatment with continuous positive airway pressure is not effective in patients with sleep apnea but no daytime symptoms. A randomised controlled trial. Ann Intern Med 2001; 134: 1015-1023.
17 Dimsdale JE, Loredo JS, Profant J. Effect of continuous positive airway pressure on blood pressure: a placebo trial. Hypertension 2000; 35: 144-147.

18 Monasterio C, Vidal S, Duran J, et al. Effectiveness of continuous positive airway pressure in mild sleep apneahypopnoea syndrome. Am J Respir Crit Care Med 2001; 164: 939-943.

19 Hedner J, Darpo B, Ejnell H, et al. Reduction in sympathetic activity after long-term CPAP treatment in sleep apnoea: cardiovascular implications. Eur Respir J 1995; 8: 222-229.

20 Jennum P, Wildschiodtz G, Christensen NJ, et al. Blood pressure, catecholamines, and pancreatic polypeptide in obstructive sleep apnea with and without nasal continuous positive airway pressure (nCPAP) treatment. $A m J$ Hypertens 1989; 2: 847-852.

21 Scottish Intercollegiate Guidelines Network. Management of obstructive sleep apnoea/hypopnoea syndrome in adults. June 2003.

22 Hedner J, Grote L. The link between sleep apnea and cardiovascular disease: time to target the non sleepy sleep apneics? Am J Resp Crit Care Med 2001; 163: 5-6.

23 Kiely JL, McNicholas WT. Cardiovascular risk factors in patients with obstructive sleep apnoea syndrome. Eur Respir J 2000; 16: 128-133.

24 MacMahon S, Peto R, Cutler J, et al. Blood pressure, stroke, and coronary heart disease. Part 1, prolonged differences in blood pressure: prospective observational studies corrected for the regression dilution bias. Lancet 1990; 335: 765-774.

25 Johns MW. A new method for measuring daytime sleepiness: the Epworth sleepiness scale. Sleep 1991; 14: 540-545.

26 Pitson DJ, Stradling JR. Autonomic markers of arousal during sleep in patients undergoing investigation for obstructive sleep apnoea, their relationship to EEG arousals, respiratory events and subjective sleepiness. J Sleep Res 1998; 7: 53-59.

27 Bennett LS, Langford BA, Stradling JR, et al. Sleep fragmentation indices as predictors of daytime sleepiness and nCPAP response in obstructive sleep apnea. Am J Respir Crit Care Med 1998; 158: 778-786.

28 Stradling JR, Smith D, Radulovacki M, et al. Effect of ondansetron on moderate obstructive sleep apnoea, a single night, placebo-controlled trial. J Sleep Res 2003; 12 : 169-170.

29 O'Brien E, Mee F, Atkins N, et al. Accuracy of the Takeda TM2420/TM-2020 determined by the British Hypertension Society protocol. J Hypertens 1991; 158: 571-572.

30 Bennett LS, Stradling JR, Davies RJ. A behavioural test to assess daytime sleepiness in obstructive sleep apnoea. $J$ Sleep Res 1997; 6: 142-145.

31 Ayas NT, Patel SR, Malhotra A, et al. Auto-titrating versus standard continuous positive airway pressure for the treatment of obstructive sleep apnea: results of a metaanalysis. Sleep 2004; 15: 249-253.

32 Moher D, Schulz KF, Altman DG, et al. The CONSORT statement: revised recommendations for improving the quality of reports of parallel randomised trials. Lancet 2001; 357: 1191-1194.

33 Parish JM, Somers VK. Obstructive sleep apnea and cardiovascular disease. Mayo Clin Proc 2004; 79: 1036-1046. 
34 Cooper VL, Bowker CM, Pearson SB, et al. Effects of simulated obstructive sleep apnoea on the human carotid baroreceptor-vascular resistance reflex. J Physiol 2004; 557: 1055-1065.

35 Rees K, Spence DP, Earis JE. Arousal responses from apneic events during non-rapid-eye-movement sleep. Am J Respir Crit Care Med 1995; 152: 1016-1021.

36 Kimoff RJ, Makino H, Horner RL, et al. Canine model of obstructive sleep apnea: model description and preliminary application. J Appl Physiol 1994; 76: 1810-1817.
37 Brooks D, Horner RL, Kimoff RJ, et al. Effect of obstructive sleep apnea versus sleep fragmentation on responses to airway occlusion. Am J Respir Crit Care Med 1997; 155: 1609-1617.

38 Brooks D, Horner RL, Kozar LF, et al. Obstructive sleep apnoea as a cause of systemic hypertension. Evidence from a canine model. J Clin Invest 1997; 99: 106-109.

39 Fletcher EC, Bao G. The rat as a model of chronic recurrent episodic hypoxia and effect upon systemic blood pressure. Sleep 1996; 19: Suppl. 10, S210-S212. 